\title{
Automatic Fingerprints Image Generation Using Evolutionary Algorithm
}

\author{
Ung-Keun Cho, Jin-Hyuk Hong, and Sung-Bae Cho \\ Dept. of Computer Science, Yonsei University \\ Biometrics Engineering Research Center \\ 134 Sinchon-dong, Seodaemun-ku \\ Seoul 120-749, Korea \\ \{bearoot, hjinh\}@sclab.yonsei.ac.kr, sbcho@cs.yonsei.ac.kr
}

\begin{abstract}
Constructing a fingerprint database is important to evaluate the performance of an automatic fingerprint recognition system. Because of the difficulty in collecting fingerprint samples, there are only few benchmark databases available. Moreover, various types of fingerprints should be required to get a fair assessment on how robust the system is against various environments. This paper presents a novel method that generates various fingerprint images automatically from only a few training samples by using the genetic algorithm. Fingerprint images generated by the proposed method include similar characteristics of those collected from the corresponding real environment. Experiments with real fingerprints verify the usefulness of the proposed method.
\end{abstract}

Keywords: fingerprint identification performance evaluation, genetic algorithm, image filtering, image generation.

\section{Introduction}

Due to the persistence and individuality of fingerprints, the fingerprint recognition has become a popular personal identification technique [1]. Recently, people consider it important to evaluate the robustness of those systems for practical application [2]. Performance evaluation, mostly dependent on benchmark databases, is a difficult process, because of the lack of public fingerprint databases involving large samples. Except for some popular fingerprint databases such as NIST database [3] and FVC databases [2], researchers usually rely on a small-scale database collected by themselves to evaluate their system. The construction of fingerprint databases requires an enormous effort so as to be incomplete, costly and unrealistic [4]. Moreover, the database should include samples collected from various environments in order to estimate the robustness of the system under realistic applications [5].

In order to measure the performance of fingerprint recognition systems from various points of view, researchers proposed several performance evaluation protocols and databases. Jain, et al. developed a twin-test by measuring the similarity of identical twins' fingerprints [6], while Pankanti, et al. theoretically estimated the individuality of fingerprints [1]. Hong, et al. reviewed performance evaluation for 
biometrics systems including fingerprint verification systems [7]. Khanna and Weicheng presented benchmarking results using NIST special database 4 [3], and Maio, et al. initiated several competitions of fingerprint verification such as FVC [2]. Simon-Zorita, et al. collected MCYT Fingerprint Database in consideration of position variability control and image quality [5].

There were some works on the generation of synthetic images for constructing databases with little cost and effort. FaceGen [9] is a modeler for generating and manipulating human faces. It manages shape, texture, expression, phones, and accessories (hair, glasses), and it can also reconstruct 3D face from single images and were used to test face recognition techniques [10]. Cappelli, et al. developed a software (SFinGE) [4] that heuristically generated fingerprint images according to some parameters, where the synthetic databases were used as one of benchmark databases in FVC [2].

In this paper, we propose a novel method that generates fingerprint images from only a few initial samples, in which the images get similar characteristics to ones manually collected from the corresponding real environment. When a target environment is given, the proposed method constructs a set of filters that modifies an original image so as to become similar to that collected in the environment. A proper set of filters is found by the genetic algorithm [8,11], where the fitness evaluation is conducted using various statistics of fingerprints to measure the similarity.

\section{Proposed Method}

\subsection{Overview}

The proposed method works similar to that of a simple genetic algorithm as shown in Fig. 1, and fitness evaluation optimizes a filter set that generates fingerprint images corresponding to a given environment. In the initialization step are set the parameters for the genetic algorithm including the population size, the maximum number of generations, the length of chromosomes, selection strategy, selection rate, crossover rate and mutation rate. The length of chromosomes means the size of a filter composed, where each gene in the chromosome represents the corresponding filter in the pool of filters. A target environment should be also determined in initialization so that the proposed method can generate images having the similar characteristics of fingerprints collected by that environment. Only a few samples are required to calculate several statistics for the target environment to evaluate a chromosome.

After initializing population, the proposed method iteratively conducts fitness evaluation, selection, crossover and mutation until a terminal condition is satisfied, in which the last 3 steps work the same as the genetic algorithm. Especially, the fitness of a chromosome is estimated according to the similarity between a few real images from the target environment and images generated after filtering. The value of each gene means a filter to apply for images of the training database. If we collect some samples from an environment which we target, the proposed method automatically analyzes the environment and finds out a set of proper filters without any expert knowledge. 


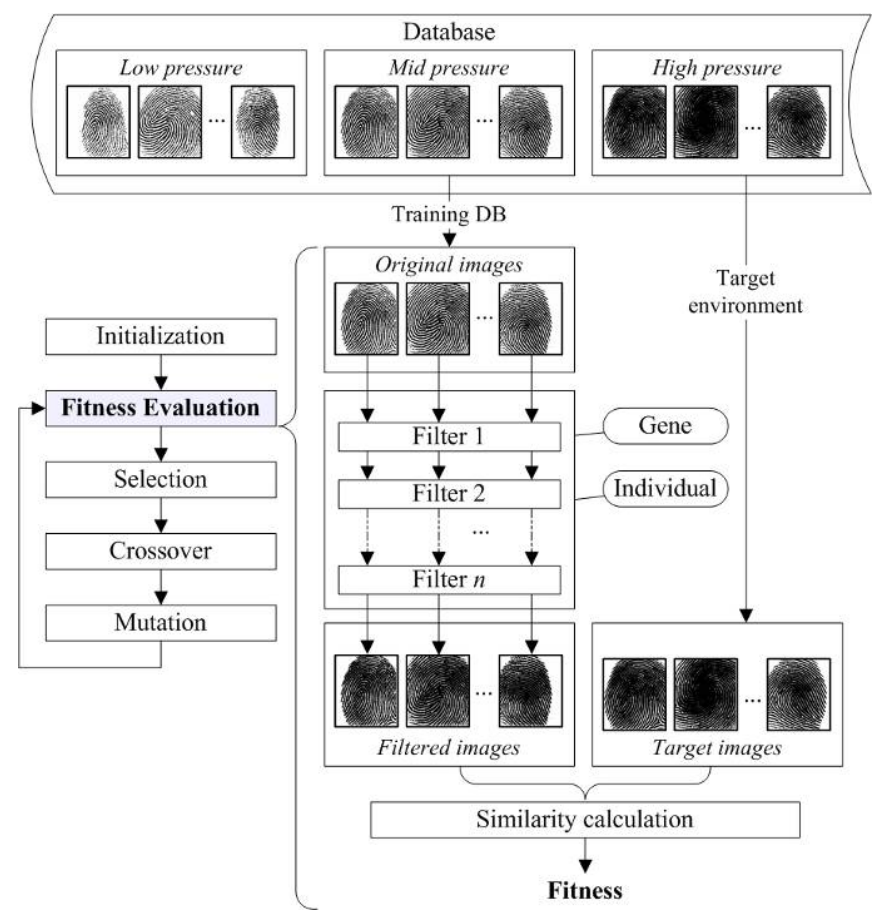

Fig. 1. Overview of the proposed method

\subsection{Image Filter Pool}

Popular image filters are used to produce similar effects from real environments. Even though each filter has a simple effect as shown in Table 1, they might produce various results when appropriately compounding with each other. The order and type of filters used in the filter set are determined by the genetic algorithm, because it is practically impossible to test all the cases of composition.

Table 1 shows the description of image filters used in this paper. They are widely used to reduce noises, smooth images, or stress the focus of images [12]. Typically, there are several categories of image filters such as histogram-based filters, mask filters and morphological filters. Various parameters like mask types make the effect of filtering more diverse. Total 70 filters construct the pool of filters.

Table 1. Image filters used in this paper

\begin{tabular}{ccc}
\hline Group & Filter & Index \\
\hline Histogram & Brightness (3 values), Contrast (3 values), & $1 \sim 9$ \\
Mask & Stretch, Equalize, Logarithm \\
Blur (6 masks), Sharper (4 masks), Median (10 & masks) & $10 \sim 29$ \\
Morphology(10 masks) & Erosion, Dilation, Opening, Closing & $30 \sim 69$ \\
& None & 0 \\
\hline
\end{tabular}




\subsection{Fitness Evaluation}

The fitness of a filter set is estimated by measuring the similarity between fingerprints collected from the target environment and images generated by the composite filter. Several representative features of fingerprints, such as the mean and variance of images, directional contrasts [13], average ridge thickness and interval [14], singularities [13] and minutiae [1], are used to design the fitness evaluation function. As mentioned before, fingerprints are easily affected by an input environment, where the statistics of fingerprints obtained might manifest the environment. Table 2 shows the features of fingerprint images and the reason to use these features for evaluating the fitness.

Table 2. Features of fingerprint images for fitness evaluation

\begin{tabular}{|c|c|c|}
\hline Feature & Description & Purpose \\
\hline Mean & The mean of gray values & Measurement of whole gray level \\
\hline Variance & The variance of gray values & Uniformity of gray values \\
\hline $\begin{array}{l}\text { Directional } \\
\text { contrast }\end{array}$ & $\begin{array}{c}\text { The mean of block directional } \\
\text { difference }\end{array}$ & $\begin{array}{c}\text { Distinctness between ridges and } \\
\text { valleys }\end{array}$ \\
\hline Thickness & The mean of ridge thickness & Measurement of ridge thickness \\
\hline Interval & The mean of valley thickness & Measurement of valley thickness \\
\hline Singularity & $\begin{array}{l}\text { The region of discontinuous } \\
\text { directional field }\end{array}$ & Major features of fingerprints \\
\hline Minutiae & $\begin{array}{c}\text { Ending and bifurcation point of } \\
\text { ridges }\end{array}$ & $\begin{array}{l}\text { Major features of fingerprint } \\
\text { recognition }\end{array}$ \\
\hline
\end{tabular}

There are 4 directional contrasts obtained by estimating the block directional difference in 8 cardinal directions without regard for the opposite direction.

Singularity is detected by the Poincare index [13], which is a popular method to compute core and delta points based on the orientation field. According to the result by the algorithm and human experts, 3 types of singularities are defined as follows:

- Missing singularity: A set of real singularities which the algorithm cannot detect.

- Spurious singularity: A set of points which the algorithm detects but are not real.

- Paired singularity: A set of real singularities which the algorithm detects well.

Minutiae points are extracted through the process of Gabor filtering, binarization and thinning the ridges [1]. Minutiae including ending and bifurcation and nothing can be detected by the algorithm and identified by human experts, respectively, so as to define 8 types of combinations as follows: ending-ending, ending-bifurcation, ending-nothing, bifurcation-ending, bifurcation-bifurcation, bifurcation-nothing, nothing-ending, nothing-bifurcation.

With these various statistics for fingerprints, the fitness evaluation function, in which weights are heuristically determined, is defined as follows. The statistics of the 
target environment is calculated from the environment database. All the values are normalized from 0 to 1 .

$$
\begin{aligned}
& \text { fitness }(i)=w_{1} \times\left(\text { mean }_{i}-\text { mean }_{\text {target }}\right) \\
& +w_{2} \times\left(\text { variance }_{i}-\text { variance }_{\text {target }}\right) \\
& +w_{3} \times \sum_{j=1}^{4}\left(\text { contrast }_{i}^{j}-\text { contrast }_{\text {target }}^{j}\right) \\
& +w_{4} \times\left(\text { thickness }_{i}-\text { thickness }_{\text {target }}\right) \\
& +w_{5} \times\left(\text { interval }_{i}-\text { interval }_{\text {target }}\right) \\
& +w_{6} \times \sum_{c \in \text { singularityType }}\left(\text { singularity }_{i}(c)-\text { singularity }_{\text {target }}(c)\right) \\
& +w_{7} \times \sum_{c \in \text { minutiaeType }}\left(\text { minutiae }_{i}(c)-\text { minutiae }_{\text {target }}(c)\right)
\end{aligned}
$$

\section{Experimental Results}

\subsection{Experimental Environment}

The usability of the proposed method is verified by comparing the fingerprints collected from real environments with those generated. A fingerprint database, used in this work, is constructed by Computer Vision Laboratory in Inha University, in which three fingerprints images were captured from each finger according to the input pressure (high (H), middle (M) and low (L)) [15]. Forty two fingerprint images of fourteen fingers are used for the training data, while forty five fingerprint images of fifteen fingers are for the test data. In the experiment, we aim to generate the fingerprints of high and low pressures from those of middle pressure. With the training data, two filter sets $(M \rightarrow H, M \rightarrow L)$ are evolved by the proposed method for each environment. Real fingerprints of high and low pressures in the training data are used to calculate the statistics of target environments as shown in Fig. 1. After evolution, the test data is used to estimate the performance of the proposed method by measuring the similarity between the real fingerprints of high and low pressures and those generated by the filters from fingerprints of middle pressure.

Fig. 2 shows the distribution of features for the training data. The input pressure affects the value of fingerprint features, which might also influence the performance of fingerprint recognition. The ridges of highly pressed fingerprints are easily connected so as to produce spurious bifurcation points, while fingerprints of low pressure are apt to generate spurious ending points. It is natural that the thickness and interval of ridges are divided to the input pressure. On the other side, singularity is less affected by the input pressure since it is calculated by the global feature like orientation. The fingerprints, collected in the environment of middle input pressure, show good performance in extracting minutiae points rather than the others. Especially, 'real ending-extracted bifur' and 'real bifur-extracted ending' of minutiae strongly show the trend of effects of the input pressure. 

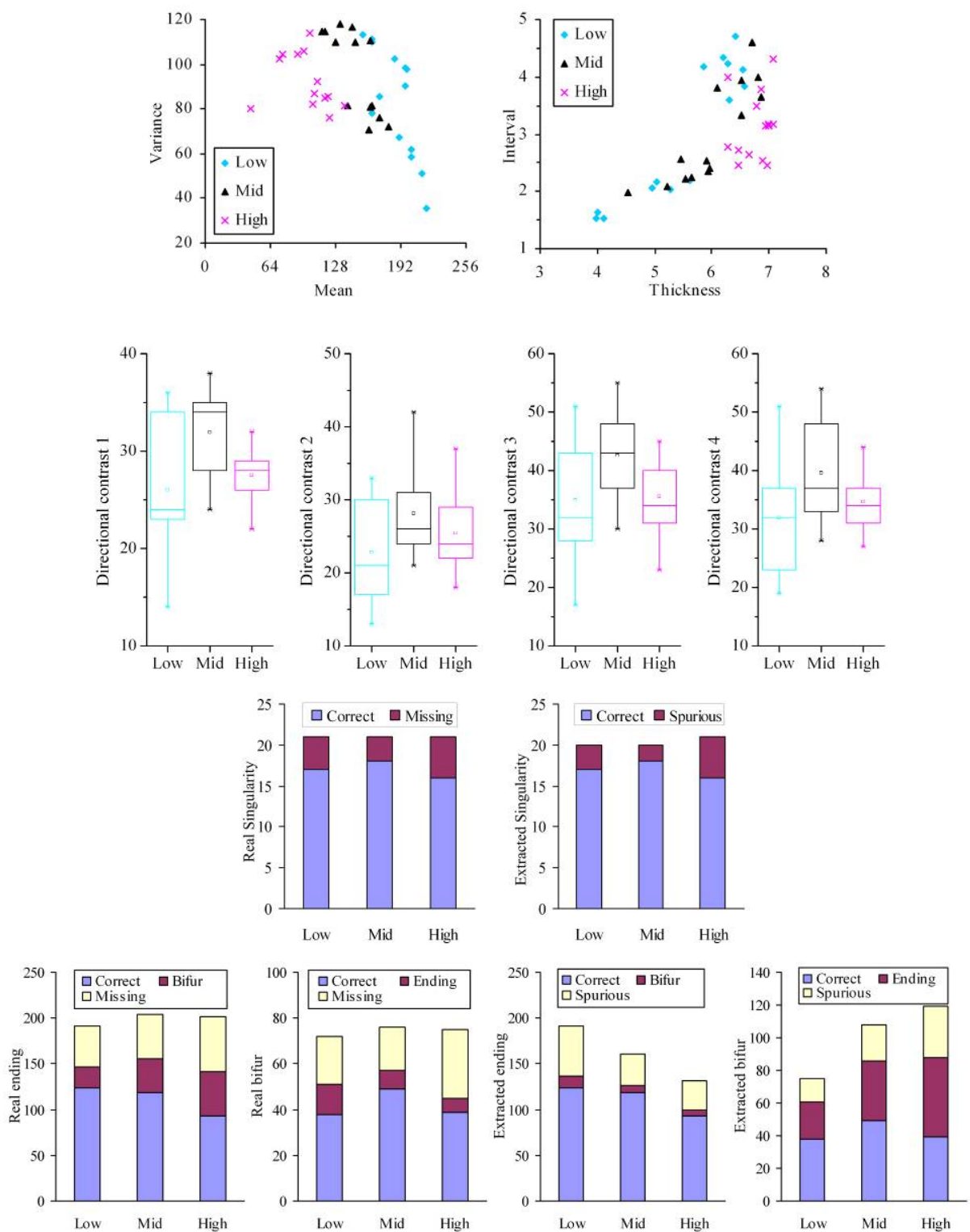

Fig. 2. Minutiae analysis of the training data

\subsection{Analysis of the Process of Evolution}

Parameters of the genetic algorithm in the experiment are set as follows: 100 generations, 50 populations, 5 gene lengths, 0.7 selection rate, 0.7 crossover rate and 0.05 mutation rate. At most five filters are used to compose a filter set because the size of chromosomes is set as five. Roulette-wheel selection is used as a basic 

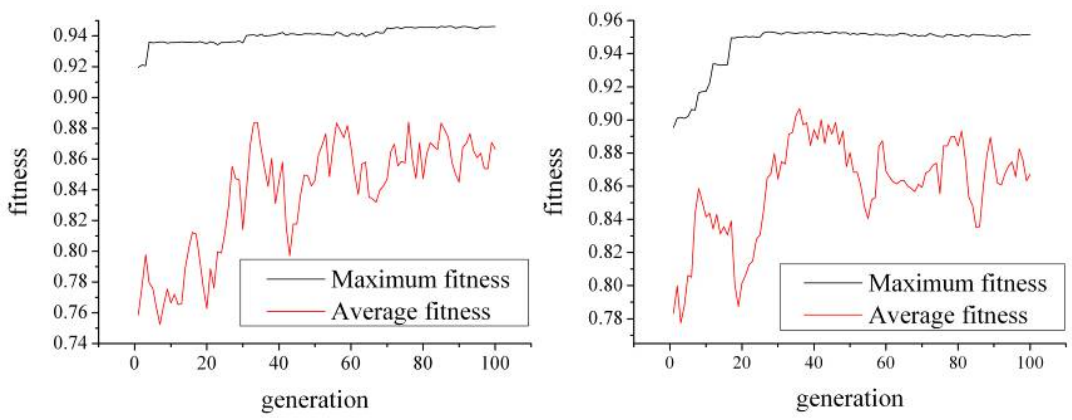

Fig. 3. Fitness through evolution (left: high pressure, right: low pressure)
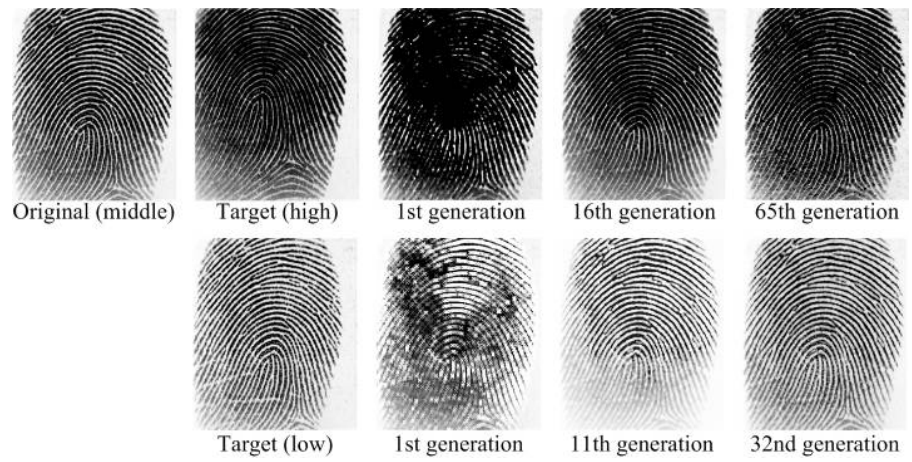

Fig. 4. Fingerprints produced by the proposed method

Table 3. Filter sets obtained through evolution

\begin{tabular}{|c|c|c|c|c|c|}
\hline Environment & & & Filter typ & & \\
\hline High & $\begin{array}{c}\text { Highpass } \\
3 \times 3 \text { \#2 }\end{array}$ & $\begin{array}{c}\text { Erosion } \\
1 \times 3\end{array}$ & $\begin{array}{c}\text { Closing } \\
3 \times 1\end{array}$ & $\begin{array}{c}\text { Closing } \\
\text { Rectangle } \\
3 \times 3\end{array}$ & None \\
\hline Low & None & None & Stretch & $\begin{array}{l}\text { Dilation } \\
\text { Diamond } \\
3 \times 3\end{array}$ & None \\
\hline
\end{tabular}

selection mechanism. Weights used in the fitness function are set as $(1,1,1,2,2,1,3)$, since the ridge information is highly affected by the input pressure.

Better filter sets are obtained by the proposed method through evolution as shown in Fig. 3. The maximum and average fitness increase as the generation grows for both target environments. Fig. 4 shows the resulting fingerprints that show similar figures to those collected from the target environments, while table 3 presents the best filter sets obtained in the last generation. 

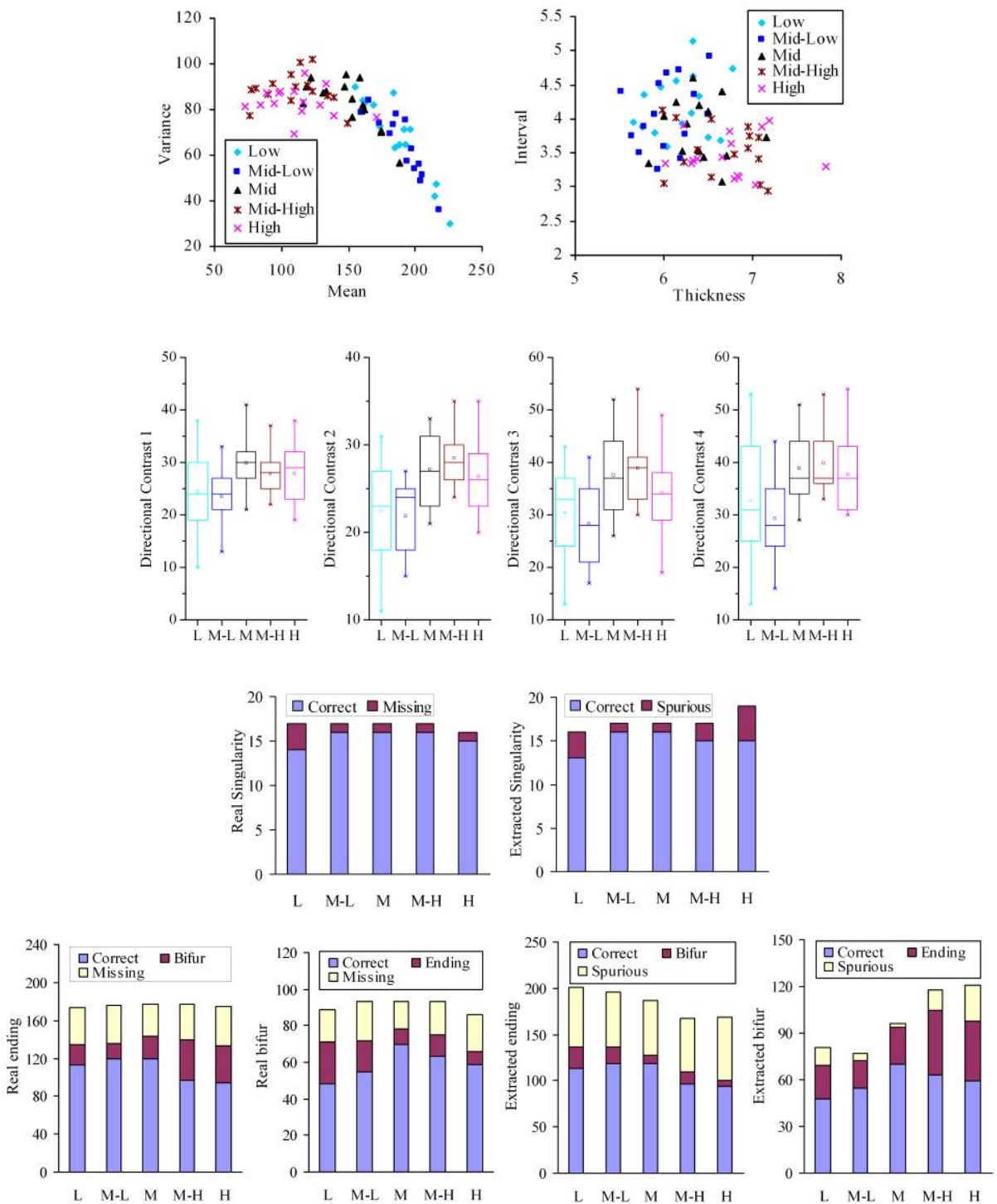

Fig. 5. Minutiae analysis of the test data

\subsection{Analysis of Generated Fingerprints}

We have analyzed the resulting fingerprints by comparing with fingerprints collected from the target environment. As shown in Fig. 5, the statistics of the fingerprints of middle pressure has been changed to be close to those calculated from the target environments, especially for mean, directional contrasts, ridge thickness and interval. Since singularity is hardly dependent on the orientation of original images, however, 
it is not able to correctly model the target environment. Fig. 5 shows that the proposed method is very useful in the distribution of minutiae extracted, where the generated fingerprints show similar aspect to those of the target environments in most cases of extracting minutiae points. Generated-high and generated-low signify the results of the proposed method.

Fig. 6 shows the impostor/genuine distribution and FMR/FNMR curves [4] on collected and generated fingerprint databases using VeriFinger 4.2 SDK. Although they are the same source fingerprints, generated images have lower performance than originals and little difference from those in the target environment.

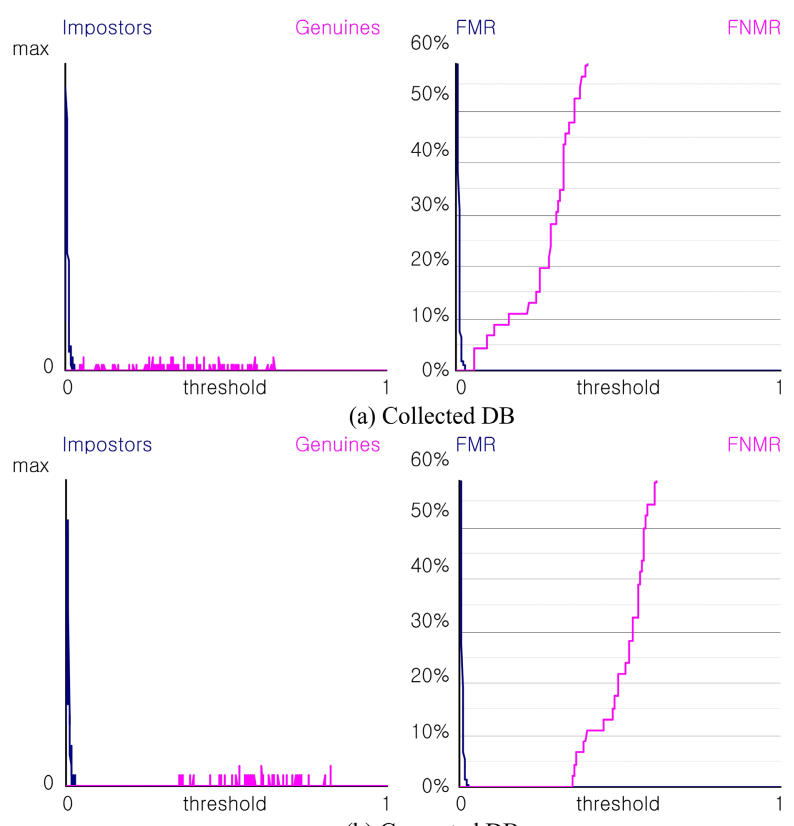

(b) Generated DB

Fig. 6. Impostor/genuine distribution and FMR/FNMR curves

\section{Concluding Remarks}

In this paper, we have proposed a novel method that automatically generates fingerprint images by using the genetic algorithm. Various simple image filters are used to construct a composite filter where the genetic algorithm searches their proper types and order. We have conducted experiments on the real database collected according to the input pressure, where the fingerprints generated by the proposed method showed the similar characteristics to those collected from real environments in terms of various statistics of fingerprint images. The generated images might be used to evaluate the performance of fingerprint recognition systems. Moreover, the proposed method has the applicability to the fingerprint image enhancement by modifying the fitness evaluation module. 
As the future work, in order to generate realistic fingerprints more precisely, we will develop a fingerprint model that characterizes them with various measures. Heuristic filters that deform fingerprints might be used to include various effects into them.

Acknowledgments. This work was supported by the Korea Science and Engineering Foundation (KOSEF) through the Biometrics Engineering Research Center (BERC) at Yonsei University.

\section{References}

[1] Pankanti, S., Prabhakar, S., Jain, A.: On the individuality of fingerprints. IEEE Trans. Pattern Analysis and Machine Intelligence 24(8), 1010-1025 (2002)

[2] Cappelli, R., Maio, D., Maltoni, D., Wayman, J.L., Jain, A.K.: Performance evaluation of fingerprint verification systems. IEEE Trans. Pattern Analysis and Machine Intelligence 28(1), 3-18 (2006)

[3] Khanna, R., Weicheng, S.: Automated fingerprint identification system (AFIS) benchmarking using the National Institute of Standards and Technology (NIST) Special Database 4. In: Proc. 28th Int. Carnahan Conf. on Security Technology, pp. 188-194 (1994)

[4] Maltoni, D.: Generation of Synthetic Fingerprint Image Databases. In: Ratha, N., Bolle, R. (eds.) Automatic Fingerprint Recognition Systems, Springer, Heidelberg (2004)

[5] Simon-Zorita, D., Ortega-Garcia, J., Fierrez-Aguilar, J., Gonzalez-Rodriguez, J.: Image quality and position variability assessment in minutiae-based fingerprint verification. IEEE Proc. Vision, Image Signal Process 150(6), 402-408 (2003)

[6] Jain, A., Prabhakar, S., Pankanti, S.: On the similarity of identical twin fingerprints. Pattern Recognition 35(11), 2653-2663 (2002)

[7] Hong, J.-H., Yun, E.-K., Cho, S.-B.: A review of performance evaluation for biometrics systems. Int. J. Image and Graphics 5(2), 501-536 (2005)

[8] Goldberg, D.: Genetic Algorithm in Search, Optimization and Machine Learning. Addison Wesley, Reading (1989)

[9] Blanz, V., Vetter, T.: A Morphable Model for the Synthesis of 3D Faces. In: Proceedings of Computer Graphics SIGGRAPH, pp. 187-194 (1999)

[10] Orlans, N., Piszcz, A., Chavez, R.: Parametrically controlled synthetic imagery experiment for face recognition testing. In: Proc. of the 2003 ACM SIGMM workshop on Biometrics methods and applications, pp. 58-64 (2003)

[11] Cho, U.-K., Hong, J.-H., Cho, S.-B.: Evolutionary singularity filter bank optimization for fingerprint image enhancement. In: Rothlauf, F., Branke, J., Cagnoni, S., Costa, E., Cotta, C., Drechsler, R., Lutton, E., Machado, P., Moore, J.H., Romero, J., Smith, G.D., Squillero, G., Takagi, H. (eds.) EvoWorkshops 2006. LNCS, vol. 3907, pp. 380-390. Springer, Heidelberg (2006)

[12] Gonzalez, R., Woods, R.: Digital Image Processing. Addison-Wesley, Reading, MA (1992)

[13] Karu, K., Jain, A.: Fingerprint Classification. Pattern Recognition 29(3), 389-404 (1996)

[14] Lim, E., Jiang, X., Yau, W.: Fingerprint quality and validity analysis. IEEE Int. Conf. on Image Processing 1, 22-25 (2002)

[15] Kang, H., Lee, B., Kim, H., Shin, D., Kim, J.: A study on performance evaluation of fingerprint sensors. In: Proc. 4th Int. Conf. Audio-and Video-based Biometric Person Authentication, pp. 574-583 (2003) 